\title{
Calculation of Electromagnetic Wave Scattering by a Small Impedance Particle of an Arbitrary Shape
}

\author{
A. G. $\operatorname{Ramm}^{1 *}$, M. I. Andriychuk ${ }^{2}$ \\ ${ }^{1}$ Department of Mathematics, Kansas State University, Manhattan, KS 66506-2602, USA \\ 2 Pidstryhach Institute for Applied Problems in Mechanics and Mathematics, NASU \\ Naukova St., 3B, 79060, Lviv, Ukraine
}

\begin{abstract}
Scattering of electromagnetic $(\mathrm{EM})$ waves by small $(k a \ll 1)$ impedance particle $D$ of an arbitrary shape, embedded in a homogeneous medium, is studied. Analytic, closed form, formula for the scattered field is derived. The scattered field is of the order $O\left(a^{2-\kappa}\right)$, where $\kappa \in[0,1)$ is a number. This field is much larger than in the case of Rayleigh-type scattering. The numerical results demonstrate a wide range of applicability of the analytic formula for the scattered field. Comparison with Mie-type solution is carried out for various boundary impedances and radii of the particle.
\end{abstract}

Keywords and phrases: electromagnetic waves, wave scattering by small body, boundary impedance, computational testing

Mathematics Subject Classification: 78A45, 65Z05

\section{Introduction}

In this paper a theory of electromagnetic (EM) wave scattering by a small impedance particle (body) $D$ of an arbitrary shape is developed. The particle is embedded in a homogeneous medium which is described by the constant permittivity $\epsilon_{0}>0$ and permeability $\mu_{0}>0$. The smallness of a particle means that $k a \ll 1$, where $a=0.5 \operatorname{diam} D$ is the characteristic dimension of a particle, $k=\omega\left(\epsilon_{0} \mu_{0}\right)^{1 / 2}$ is the wave number in the medium exterior to the particle. The approach can be easily extended to the case of many particles. Although scattering of EM waves by small bodies has a long history, going back to Rayleigh (1871), see [2], [17], the results of this paper are new and useful in applications because light scattering by colloidal particles in a solution, and light scattering by small dust particles in the air are examples of the problems to which our theory is applicable. The Mie theory deals with the EM wave scattering by a sphere, not necessarily small, and gives the solution to the scattering problem in terms of the series in spherical harmonics. If the sphere is small, $k a \ll 1$, then the first term in the Mie series yields the main part of the solution. Our theory is applicable only to small particles. They can be of an arbitrary shape. The solution to the scattering problem for one small particle of an arbitrary shape is given analytically, in closed form. Wave scattering problem in the case of one body can be studied theoretically only in the limiting cases of a small body, $k a \ll 1$, or a large body, $k a \gg 1$. In the latter case the geometrical optics is applicable. This paper deals with the case $k a \ll 1$. Rayleigh (1871) understood that the scattering by

*Corresponding author. E-mail: ramm@math.ksu.edu 
a small body is given mainly by the dipole radiation. For a small body of an arbitrary shape this dipole radiation is determined by the polarization moment, which is defined by the polarizability tensor. For homogeneous bodies of arbitrary shape analytic formulas, which allow one to calculate this tensor with any desired accuracy, were derived in [17],[18]. These bodies were assumed dielectric or conducting in [17]. Under the Rayleigh assumption the scattered field is proportional to $a^{3}$, that is, to the volume of a small body. The physically novel feature of our theory is a conclusion that for small impedance particle with the impedance $\zeta=h a^{-\kappa}$, where $h$ is a given constant, Reh $\geq 0$, and $\kappa \in[0,1)$ is a given number, the scattered field is proportional to $a^{2-\kappa}$. Therefore, the scattered field in this case is much larger than in the Rayleigh case, since $a^{3} \ll a^{2-\kappa}$ if $a \ll 1$ and $\kappa \geq 0$. This physically interesting conclusion may be of practical importance.

In this paper wave scattering by one small impedance particle is studied. Besides high intrinsic interest in this problem, the theory we develop allows one to generalize it to the case of many particles and to obtain some physically interesting conclusions about the changes of the material properties of the medium in which many small particles are embedded. These results will be presented in another paper and will be used for developing a method for creating materials with a desired refraction coefficient by embedding many small impedance particles into a given material, similarly to the results in a series of papers [4]-[15] for scalar wave scattering. The novel physical idea in this paper is to reduce solving the scattering problem to finding some constant pseudovector $Q$ (see formula (2.15)), rather than a function $J$ (see formula (2.7)) on the surface of the scatterer. The quantity $Q$ is a pseudovector which somewhat analogous to the total charge on the surface of the perfect conductor with the shape of $D$, while the function $J$ is somewhat analogous to the surface charge density in electrostatics. We assume for simplicity that the impedance $\zeta$ (see formula (2.2)) is a constant given in (2.13). A similar assumption appeared in paper [5], where scalar wave scattering theory was developed. The results of the theory in [5] was a recipe for creating materials with a desired refraction coefficient in acoustics (see [13], [14], [15]). The boundary impedance (2.13) grows to infinity as $a \rightarrow 0$. The impedance boundary condition is widely applicable in physics and does not require that the body be small or large. One can pass to the limit in the equation for the effective (self-consistent) field in the medium, obtained by embedding many small impedance particles into a given medium. Such a theory is briefly summarized in paper [14] for the scalar wave scattering, where the equation for the limiting field in the medium is derived. The aim of this paper is to develop a similar theory for the EM wave scattering by a small impedance particle embedded in a given material and to carry out the numerical calculations showing the possibility to use the developed theory in the wide range of the parameters, such as the radius $a$ of the particle, its boundary impedance $\zeta$, the distance $d$ between the particle and the observation point, and the wavelength.

For EM wave scattering by one small body $D$ of an arbitrary shape with an impedance boundary condition the following analytic formula for the electromagnetic field in the region $r:=|x| \gg a$, is derived:

$$
E(x)=E_{0}(x)+\left[\nabla \frac{e^{i k r}}{4 \pi r}, Q\right], \quad r \gg a,
$$

where $g(x, y):=\frac{e^{i k r}}{4 \pi r}, \quad r=|x-y|, E_{0}$ is the incident field, which satisfies Maxwell's equations in the absence of the scatterer $D,[A, B]=A \times B$ is the cross product of two vectors, $\left(Q, e_{j}\right)=Q \cdot e_{j}$ is the dot product, $\left\{e_{j}\right\}_{j=1}^{3}$ is an orthonormal basis in $\mathbb{R}^{3}$,

$$
Q_{j}:=\left(Q, e_{j}\right)=-\frac{\zeta|S|}{i \omega \mu_{0}} \Xi_{j p}\left(\nabla \times E_{0}(O)\right)_{p}, \quad \Xi:=(I+\alpha) \tau
$$

over the repeated index $p$ summation is understood from 1 to $3, \zeta$ is the boundary impedance, $|S|$ is the surface area of the particle, the matrix $\Xi_{j p}$ is defined by the formula

$$
\Xi_{j p}:=(I+\alpha)\left(\delta_{j p}-|S|^{-1} \int_{S} N_{j}(s) N_{p}(s) d s\right):=(I+\alpha) \tau_{j p},
$$


where $N_{j}(s)$ is the $j$-th component of the unit normal $N(s)$ to the surface $S$ at a point $s \in S$, pointing out of $D, \tau:=I-b, b$ is a matrix:

$$
b_{j p}:=|S|^{-1} \int_{S} N_{j}(s) N_{p}(s) d s
$$

$k=\omega\left(\epsilon_{0} \mu_{0}\right)^{1 / 2}$ is the wave number, $O \in D$ is the origin, $I$ is the identity matrix, and $\alpha$ is a matrix.

For small particles, $a \rightarrow 0$, one may set matrix $\alpha=0$ and get $\Xi=\tau$.

By $S^{2}$ the unit sphere in $\mathbb{R}^{3}$ is denoted. The boundary $S$ of the small body $D$ is assumed smooth. It is sufficient to assume that in the local coordinates the equation of $S$ is $x_{3}=\phi\left(x_{1}, x_{2}\right)$, where the function $\phi$ has first derivatives satisfying a Hölder condition.

The main analytical results of this paper are formulas (1.1)-(1.3) and equations (2.29) and (2.30).

The scattering problem by one small body is formulated and studied in Section 2. The numerical results demonstrating the applicability of the proposed theory to computation of the electromagnetic field in a wide range of problem's parameters are given in Section 3. In Section 4 the conclusions are formulated.

In this paper we do not solve the boundary integral equation to which the scattering problem can be reduced in a standard approach, but find an asymptotically exact closed form expression for the pseudovector $Q$, which defines the behavior of the scattered field at the distances $d \gg a$.

These distances $d$ can be very small if $a$ is sufficiently small, and $d$ can be much less than the wavelength $\lambda=\frac{2 \pi}{k}$.

\section{EM wave scattering by one small impedance particle}

Let us use in this Section the following notations: $D$ is a small body, $D^{\prime}:=\mathbb{R}^{3} \backslash D, k>0$ is a wave number, $k a \ll 1, a=0.5 \operatorname{diam} D, k=\frac{2 \pi}{\lambda}, \lambda$ is the wavelength of the incident EM wave, $k^{2}=\omega^{2} \epsilon_{0} \mu_{0}$, where $\omega$ is the frequency and $\epsilon_{0}, \mu_{0}$ are constant permittivity and permeability of the medium. Our arguments remain valid if one assumes that the medium has a constant conductivity $\sigma_{0} \geq 0$. In this case $\epsilon_{0}$ is replaced by $\epsilon_{0}+i \frac{\sigma_{0}}{\omega}$. Denote by $S$ the boundary of $D$, by $|S|$ its surface area, by $V$ the volume of $D$, by $[E, H]=E \times H$ the cross product of two vectors, and by $(E, H)=E \cdot H$ the dot product of two vectors, $N$ is the unit normal to $S$ pointing out of $D, \zeta$ is the boundary impedance of the particle.

Let $D$ be embedded in a homogeneous medium with constant parameters $\epsilon_{0}, \mu_{0}$. Electromagnetic (EM) wave scattering problem consists of finding vectors $E$ and $H$ satisfying Maxwell's equations:

$$
\nabla \times E=i \omega \mu_{0} H, \quad \nabla \times H=-i \omega \epsilon_{0} E \quad \text { in } \quad D^{\prime}:=\mathbb{R}^{3} \backslash D,
$$

the impedance boundary condition:

$$
[N,[E, N]]=\zeta[N, H] \quad \text { on } \quad S
$$

and the radiation condition:

$$
E=E_{0}+v_{E}, \quad H=H_{0}+v_{H},
$$

where $E_{0}, H_{0}$ are the incident fields satisfying equations $(2.1)$ in all of $\mathbb{R}^{3}, v_{E}:=v$ and $v_{H}$ are the scattered fields. In the literature, for example in [2], the impedance boundary condition is written sometimes as $E^{t}=\zeta\left[H^{t}, N\right]$, where $N$ is the unit normal on $S$ pointed into $D$. Since our $N$ is pointed out of $D$, our impedance boundary condition (2.2) is the same as in [2].

One often assumes that the incident wave is a plane wave, i.e., $E_{0}=\mathcal{E} e^{i k \alpha \cdot x}, \mathcal{E}$ is a constant vector, $\alpha \in S^{2}$ is a unit vector, $S^{2}$ is the unit sphere in $\mathbb{R}^{3}, \alpha \cdot \mathcal{E}=0, v_{E}$ and $v_{H}$ satisfy the Sommerfeld radiation condition: $r\left(\frac{\partial v}{\partial r}-i k v\right)=o(1)$ as $r:=|x| \rightarrow \infty$, and, consequently, $\left[r^{0}, E\right]=H+o\left(r^{-1}\right)$ and $\left[r^{0}, H\right]=-E+o\left(r^{-1}\right)$ as $r:=|x| \rightarrow \infty$, where $r^{0}:=x / r$.

It is assumed in this paper that the impedance $\zeta$ is a constant, $\operatorname{Re} \zeta \geq 0$. 
This assumption guarantees the uniqueness of the solution to Maxwell's equations satisfying the radiation condition. For completeness a proof of the uniqueness result is given in Lemma 1. The tangential component of $E$ on $S, E^{t}$, is defined as:

$$
E^{t}=E-N(E, N)=[N,[E, N]]
$$

This definition differs from the one used often in the literature, namely, from the definition $E^{t}=[N, E]$. Our definition (2.4) corresponds to the geometrical meaning of the tangential component of $E$ and, therefore, should be used. The impedance boundary condition is written in [2] as $E^{t}=\zeta\left[H^{t}, N_{i}\right]$, where $\zeta$ is the boundary impedance and $N_{i}$ is the unit normal to $S$ pointing into $D$. In our paper $N$ is the unit normal pointing out of $D$. Therefore, the impedance boundary condition in our paper is written as in equation (2.2). If one uses definition (2.4), then this condition reduces to (2.2), because $[[N,[H, N]], N]=[H, N]$. The assumption $\operatorname{Re} \zeta \geq 0$ is physically justified by the fact that this assumption guarantees the uniqueness of the solution to the boundary problem (2.1)-(2.3).

Lemma 1. Problem (2.1)-(2.3) has at most one solution.

For a proof of Lemma 1, we refer the reader to [4]. Let us note that problem (2.1)-(2.3) is equivalent to the problem

$$
\begin{gathered}
\nabla \times \nabla \times E=k^{2} E \text { in } D^{\prime}, \quad H=\frac{\nabla \times E}{i \omega \mu_{0}}, \\
{[N,[E, N]]=\frac{\zeta}{i \omega \mu_{0}}[N, \nabla \times E] \text { on } S,}
\end{gathered}
$$

together with the radiation condition (2.3). Thus, we have reduced the scattering problem to finding one vector $E(x)$. If $E(x)$ is found, then $H=\frac{\nabla \times E}{i \omega \mu_{0}}$, and the pair $E$ and $H$ solves Maxwell's equations, satisfies the impedance boundary condition and the radiation condition (2.3).

Let us look for $E$ of the form

$$
E=E_{0}+\nabla \times \int_{S} g(x, t) J(t) d t, \quad g(x, y)=\frac{e^{i k|x-y|}}{4 \pi|x-y|},
$$

where $E_{0}$ is the incident field, which satisfies Maxwell's equations in the absence of the scatterer $D, t$ is a point on the surface $S, t \in S, d t$ is an element of the area of $S$, and $J(t)$ is an unknown pseudovectorfunction on $S$, which is tangential to $S$, i.e., $N(t) \cdot J(t)=0$, where $N(t)$ is the unit normal to $S$ at the point $t \in S$. That $J=J(t)$ is a pseudovector follows from the fact that $E$ is a vector and $\nabla \times(g J)$ is a vector only if $J$ is a pseudovector, because $g$ is a scalar.

It is assumed that $J$ is a smooth function on $S$, for example, $J \in C^{2}(S)$.

The right-hand side of (2.7) solves equation (2.5) in $D$ for any continuous $J(t)$, because $E_{0}$ solves (2.5) and

$$
\begin{aligned}
& \nabla \times \nabla \times \nabla \times \int_{S} g(x, t) J(t) d t= \\
& \text { graddiv } \nabla \times \int_{S} g(x, t) J(t) d t- \\
& \nabla^{2} \nabla \times \int_{S} g(x, t) J(t) d t= \\
& k^{2} \nabla \times \int_{S} g(x, t) J(t) d t, \quad x \in D^{\prime} .
\end{aligned}
$$

Here we have used the known identity divcurl $E=0$, valid for any smooth vector field $E$, and the known formula

$$
-\nabla^{2} g(x, y)=k^{2} g(x, y)+\delta(x-y)
$$


The integral $\int_{S} g(x, t) J(t) d t$ satisfies the radiation condition. Thus, formula (2.7) solves problem $(2.5)$, (2.6), (2.3), if $J(t)$ is chosen so that boundary condition (2.6) is satisfied.

Let $O \in D$ be a point inside $D$. The following known formula (see, for example, [3]) is useful:

$$
\left[N, \nabla \times \int_{S} g(x, t) J(t) d t\right]_{\mp}=\int_{S}\left[N_{s},\left[\left.\nabla_{x} g(x, t)\right|_{x=s}, J(t)\right]\right] d t \pm \frac{J(s)}{2},
$$

where the \pm signs denote the limiting values of the left-hand side of (2.10) as $x \rightarrow s$ along the normal to $S$ at the point $s$ from $D$, respectively, from $D^{\prime}$. To derive an integral equation for $J=J(t)$, substitute $E(x)$ from (2.7) into impedance boundary condition (2.6), and get

$$
0=f+\left[N,\left[\nabla \times \int_{S} g(s, t) J(t) d t, N\right]\right]-\frac{\zeta}{i \omega \mu_{0}}\left[N, \nabla \times \nabla \times \int_{S} g(s, t) J(t) d t\right],
$$

where

$$
f:=\left[N,\left[E_{0}(s), N\right]\right]-\frac{\zeta}{i \omega \mu_{0}}\left[N, \nabla \times E_{0}\right]
$$

We assume that

$$
\zeta=\frac{h}{a^{\kappa}}
$$

where $\operatorname{Re} h \geq 0$. and $\kappa \in[0,1)$ is a constant.

The requirement $\operatorname{Re} \zeta \geq 0$ is the only physical requirement that is imposed on the boundary impedance. Our assumption (2.13) satisfies this requirement. This assumption allows us to derive the formulas (1.1) and (1.2) for the scattered field. Formulas (1.1)-(1.2) are new and of physical importance because they yield the value for the scattered field that is $O\left(a^{2-\kappa}\right)$, which is much larger than the usual Rayleigh-type estimate $O\left(a^{3}\right)$ for the field scattered by a small body of characteristic size $a$.

Let us write (2.7) as

$$
E(x)=E_{0}(x)+\left[\nabla_{x} g(x, O), Q\right]+\nabla \times \int_{S}(g(x, t)-g(x, O)) J(t) d t,
$$

where

$$
Q:=\int_{S} J(t) d t
$$

The central physical idea of the theory, developed in this paper, is: the third term in the right-hand side of (2.14) is negligible compared with the second term if $k a \ll 1$. Consequently, the scattering problem is solved if $Q$ is found. The traditional approach requires finding an unknown function $J(t)$, which is usually found numerically by the boundary integral equations (BIE) method. The reason for the third term in the right-hand side of (2.14) to be negligible compared with the second one, is explained below. In the estimates, used in this explanation, the smallness of the body is used essentially: even if one is in the far zone, that is, $\frac{a}{d} \ll 1$, one cannot conclude that estimate (2.18) (see below) holds unless one assumes that $k a \ll 1$. Thus, the third term in (2.14) cannot be neglected in the far zone if the condition $k a \ll 1$ does not hold.

We prove below that

$$
Q=O\left(a^{2-\kappa}\right)
$$

To prove that the third term in the right-hand side of (2.14) is negligible compared with the second one, let us establish several estimates valid if $a \rightarrow 0$ and $d:=|x-O| \gg a$. Under these assumptions one has

$$
j_{1}:=\left|\left[\nabla_{x} g(x, O), Q\right]\right| \leq O\left(\max \left\{\frac{1}{d^{2}}, \frac{k}{d}\right\}\right) O\left(a^{2-\kappa}\right),
$$




$$
\begin{aligned}
& j_{2}:=\left|\nabla \times \int_{S}(g(x, t)-g(x, O)) \sigma(t) d t\right| \\
& \leq a O\left(\max \left\{\frac{1}{d^{3}}, \frac{k^{2}}{d}\right\}\right) O\left(a^{2-\kappa}\right),
\end{aligned}
$$

and

$$
\left|\frac{j_{2}}{j_{1}}\right|=O\left(\max \left\{\frac{a}{d}, k a\right\}\right) \rightarrow 0, \quad \frac{a}{d}=o(1), a \rightarrow 0 .
$$

These estimates show that one may neglect the third term in (2.14), and write

$$
E(x)=E_{0}(x)+\left[\nabla_{x} g(x, O), Q\right] .
$$

The error of this formula tends to zero as $a \rightarrow 0$ under our assumptions.

Note that the inequality $|x| \gg k a^{2}$ is satisfied even for $|x|=O(a)$ if $k a \ll 1$. Thus, formula (2.19) is applicable in a wide region.

Let us estimate $Q$ asymptotically, as a $\rightarrow 0$.

Take the cross product of (2.11) with $N$ and integrate the resulting equation over $S$ to get

$$
I_{0}+I_{1}+I_{2}=0
$$

where $I_{0}$ is defined in formula (2.20) (see below), $I_{1}$ is defined in (2.23), and

$$
I_{2}:=-\int_{S} \frac{\zeta}{i \omega \mu_{0}}\left[N,\left[N, \nabla \times \nabla \times \int_{S} g(s, t) J(t) d t\right]\right] d s .
$$

Let us estimate the order of $I_{0}$ as $a \rightarrow 0$. One has

$$
I_{0}=\int_{S}\left(\left[N, E_{0}\right]-\frac{\zeta}{i \omega \mu_{0}}\left[N,\left[N, \nabla \times E_{0}\right]\right]\right) d s .
$$

In what follows we keep only the main terms as $a \rightarrow 0$, and denote by the sign $\simeq$ the terms equivalent up to the terms of higher order of smallness as $a \rightarrow 0$.

One has

$$
I_{00}:=\int_{S}\left[N, E_{0}\right] d s=\int_{D} \nabla \times E_{0} d x \simeq \nabla \times E_{0}(O) V=O\left(a^{3}\right),
$$

where $d x$ is the element of the volume, $O \in D$ is a point chosen as the origin, and $V$ is the volume of $D$.

Denoting by $|S|$ the surface area of $S$, one obtains

$$
I_{01}:=-\int_{S} \frac{\zeta}{i \omega \mu_{0}}\left[N,\left[N, \nabla \times E_{0}\right]\right] d s=\frac{\zeta|S|}{i \omega \mu_{0}} \tau \times E_{0},
$$

where

$$
\tau:=I-b, \quad b_{m j}:=\frac{1}{|S|} \int_{S} N_{m}(t) N_{j}(t) d t,
$$

$b=\left(b_{m j}\right)$ is a matrix which depends on the shape of $S$, and $I:=\delta_{m j}$ is the unit matrix. Since $\zeta=O\left(a^{-\kappa}\right)$ and $|S|=O\left(a^{2}\right)$, one concludes that $I_{01}=O\left(a^{2-\kappa}\right)$. Consequently, $\left|I_{00}\right| \ll\left|I_{01}\right|$, because $I_{00}=O\left(a^{3}\right)$ as $a \rightarrow 0$. Thus,

$$
I_{0} \simeq I_{01}=O\left(a^{2-\kappa}\right), \quad a \rightarrow 0 .
$$

Let us consider $I_{1}$ :

$$
I_{1}=\int_{S}\left(\int_{S}[N,[\nabla g(s, t), J(t)]] d t+\frac{J(s)}{2}\right) d s:=I_{11}+\frac{Q}{2} .
$$


One has

$$
I_{11}=\int_{S} d s \int_{S} d t\left(\nabla g(s, t) N(s) \cdot J(t)-J(t) \frac{\partial g(s, t)}{\partial N(s)}\right) .
$$

It is well known that $\int_{S} \frac{\partial g_{0}(s, t)}{\partial N(s)} d s=-\frac{1}{2}$, where $g_{0}:=\frac{1}{4 \pi|s-t|}$. Since

$$
g(s, t)-g_{0}(s, t)=\frac{i k}{4 \pi}+O(|s-t|), \quad|s-t| \rightarrow 0,
$$

and $|s-t|=O(a)$, one concludes that if $a \rightarrow 0$ then

$$
\int_{S} \frac{\partial g(s, t)}{\partial N(s)} d s \simeq-\frac{1}{2}
$$

and

$$
\int_{S} d t \int_{S} d s \nabla g(s, t) N(s) \cdot J(t):=O\left(a^{3}\right),
$$

because $|N(s) \cdot J(t)|=O(|s-t|)$ as $|s-t| \rightarrow 0, \nabla g=O\left(\frac{1}{|s-t|^{2}}\right)$, and

$$
\int_{S} d t \int_{S} d s|s-t|^{-1}=O\left(a^{3}\right)
$$

Therefore,

$$
I_{1} \simeq Q
$$

Matrix $b$ for spheres is calculated at the end of Section 2. The estimate $N(s) \cdot J(t)=O(|s-t|)$ as $|s-t| \rightarrow 0$ holds because $J$ is tangential to $S$ and this implies the estimate $\cos \theta(s, t)=O(|s-t|)$, where $\theta(s, t)$ is the angle between $N(s)$ and $J(t)$.

Let us show that $I_{2}=O\left(a^{4-\kappa}\right)$ and therefore $I_{2}$ is negligible compared with $I_{0}$ as $a \rightarrow 0$. If this is done, then the equation for $Q$ is

$$
Q=-\frac{\zeta|S|}{i \omega \mu_{0}} \tau \nabla \times E_{0}
$$

From (2.28) it follows that

$$
E(x)=E_{0}(x)-\frac{\zeta|S|}{i \omega \mu_{0}}\left[\nabla_{x} g(x, O), \tau \nabla \times E_{0}(O)\right] .
$$

This equation is our first main result which gives an analytic formula for the solution of the EM wave scattering problem by a small body of an arbitrary shape, on the boundary of which an impedance boundary condition holds.

In the far zone $r:=|x| \rightarrow \infty$ one has $\nabla_{x} g(x, O)=i k g(x, O) x^{0}+O\left(r^{-2}\right)$, where $x^{0}:=x / r$ is a unit vector in the direction of $x$. Consequently, for $r \rightarrow \infty$ one can rewrite formula (2.29) as

$$
E(x)=E_{0}(x)-\zeta|S|\left(\frac{\epsilon_{0}}{\mu_{0}}\right)^{1 / 2} \frac{e^{i k r}}{r}\left[x^{0}, \tau \nabla \times E_{0}(O)\right] .
$$

This field is orthogonal to the radius-vector $x$ in the far zone as it should be.

Let us show that the term $I_{2}$ is negligible as $a \rightarrow 0$. Remember that curlcurl $=$ graddiv $-\nabla^{2}$ and $-\nabla^{2} g(x, t)=k^{2} g(x, t)+\delta(x-y)$. Therefore,

$$
-\nabla^{2} \int_{S} g(x, t) J(t) d t=k^{2} \int_{S} g(x, t) J(t) d t .
$$

Consequently,

$$
-\left.i \omega \mu_{0} I_{2} \simeq \zeta \int_{S} d s\left[N,\left[N, \operatorname{graddiv} \int_{S} g(x, t) J(t) d t\right]\right]\right|_{x \rightarrow s}:=I_{21}
$$


Since the function $J(t)$ is assumed smooth, one has

$$
\operatorname{div} \int_{S} g(x, t) J(t) d t=\int_{S} g(x, t) \operatorname{div} J(t) d t, \quad \operatorname{div} J=\frac{\partial J_{m}}{\partial t_{m}}
$$

summation is understood here (and below) over the repeated indices.

Furthermore,

$$
\operatorname{grad} \int_{S} g(x, t) \frac{\partial J_{m}}{\partial t_{m}} d t=e_{p} \int_{S} g(x, t) \frac{\partial^{2} J_{m}}{\partial t_{p} \partial t_{m}} d t
$$

where the relation $\frac{\partial g(x, t)}{\partial x_{p}}=-\frac{\partial g(x, t)}{\partial t_{p}}$ was used, and an integration by parts with respect to $t_{p}$ has been done over the closed surface $S$. Therefore

$$
I_{2} \leq c|\zeta| \int_{S} d s \int_{S}|g(s, t)|=O\left(a^{4-\kappa}\right) \ll I_{0} .
$$

The constant $c>0$ here is a bound on the second derivatives of $J$ on $S$.

Example of calculation of matrix $b$. Let us calculate $b$ for a sphere of radius $a$ centered at the origin. One has:

$$
b_{j m}=\frac{1}{|S|} \int_{S} N_{j}(t) N_{m}(t) d t=\frac{1}{3} \delta_{j m} .
$$

Note that $|S|=4 \pi a^{2}, d t=a^{2} \sin \theta d \theta d \phi$, and $N_{m}(t)$ is proportional to the spherical harmonic $Y_{1, m}$, so the above formula for $j \neq m$ follows from the orthogonality properties of the spherical harmonics, and for $m=j$ this formula is a consequence of the normalization $|N|=1$.

Let us formulate the result of this Section as a theorem.

Theorem 1. If $k a \ll 1$, then the solution to the scattering problem (2.1)-(2.3) is given by formula (2.29).

In Section 3 numerical calculations of the EM waves, scattered by a small body (particle), are carried out for a variety of the problem's parameters.

\section{Numerical results}

In paper [1] some numerical results were obtained on the comparison of the asymptotic solution, found in [19], and the Mie-type solution given in [1]. The asymptotic solution from [1] was compared with the Mie-type solution in far zone. In Section 2 analytic formula (2.29) for the EM field everywhere outside an immediate neighborhood of the small particle was given, and formula (2.30) for this field in the far zone was derived.

It is of practical interest to evaluate the accuracy of formula (2.30) and compare it with the accuracy of formula (2.29). It is also of practical interest to find out for which values of the problem parameters these two formulas differ from the Mie-type solution by not more than $5 \%-10 \%$. To compare the asymptotic solution (2.30) with the Mie-type solution one should establish the range of applicability of the asymptotic formula (2.30) and compare the results, obtained by this formula, with the results obtained by formula (2.29) for a variety of the values of the parameters $a, r$, and $\zeta$, where $r$ is distance to the far zone, and $\zeta$ is the boundary impedance. Let us rewrite formulas (2.29) and (2.30) in the form, suitable for numerical calculations. To do this, let us use the following formulas for $\nabla_{x} g(x, O)$ and $\nabla_{x} E_{0}(O)$ :

$$
\nabla_{x} g(x, O)=\nabla_{x} \frac{e^{i k|x|}}{4 \pi|x|}=\frac{e^{i k|x|}}{4 \pi|x|^{2}}\left(i k-\frac{1}{|x|}\right) x
$$

and

$$
\nabla \times E_{0}(O)=\nabla \times\left(\begin{array}{c}
e^{i k x_{3}} \\
0 \\
0
\end{array}\right)_{\mid x=0}=\left(\begin{array}{c}
0 \\
i k \\
0
\end{array}\right)
$$




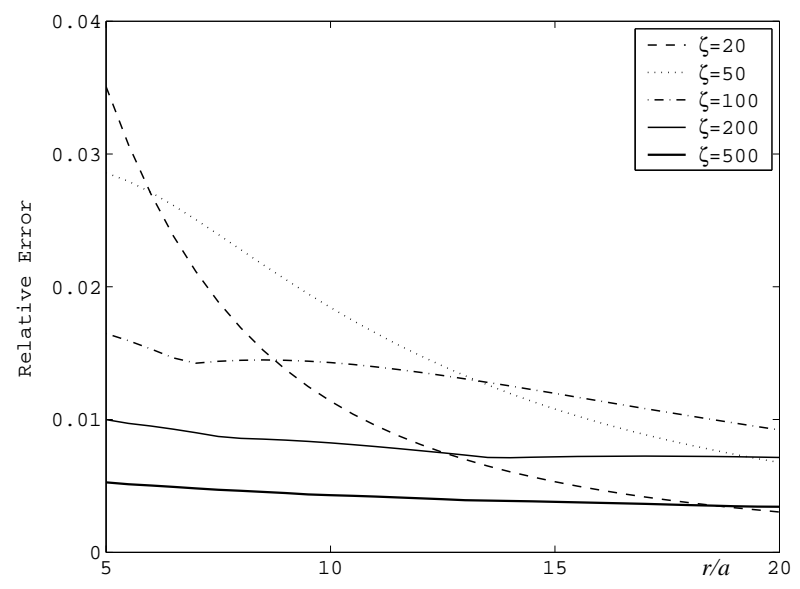

FiguRE 1. Relative error for $E_{1}$ component $k=0.001$

Parameter $\tau=2 / 3$ because $b_{j m}=\frac{1}{3} \delta_{j m}$. Using these formulas and formulas (3.1) and (3.2), one gets the following representation for the EM field:

$$
E(x)=e^{i k x_{3}}\left(\begin{array}{l}
1 \\
0 \\
0
\end{array}\right)-\frac{8 a^{2} \pi k \zeta}{3 \omega \mu_{0}} \frac{e^{i k|x|}}{4 \pi|x|^{2}}\left(i k-\frac{1}{|x|}\right)\left(\begin{array}{c}
-x_{3} \\
0 \\
x_{1}
\end{array}\right),
$$

and the corresponding formula valid in the far zone,

$$
E(r)=e^{i k x_{3}}\left(\begin{array}{l}
1 \\
0 \\
0
\end{array}\right)-\frac{8 a^{2} \pi \zeta}{3} \sqrt{\frac{\varepsilon_{0}}{\mu_{0}}} \frac{e^{i k r}}{r} i k\left(\begin{array}{c}
-x_{3}^{0} \\
0 \\
x_{1}^{0}
\end{array}\right),
$$

where $x_{1}^{0}=x_{1} / r, x_{3}^{0}=x_{3} / r$ are the unit vectors in the directions $x_{1}$ and $x_{3}$.

\subsection{Relative error of formula (3.4) for far zone}

The dependence of relative error of the asymptotic solution (3.4) on the parameter $r / a$ is investigated here. In Figs. 1-3 the numerical results demonstrate the relative error of asymptotic formula (3.4) for the $E_{1}, E_{2}$, and $E_{3}$ components for various values of the boundary impedance $\zeta$, when $k=0.001$ and $a=1.0$. The relative error is calculated by formula

$$
R E=\frac{\left|S_{(3.3)}-S_{(3.4)}\right|}{\left|S_{(3.3)}\right|}
$$

where $S_{(3.3)}$ is the exact solution by formula (3.3), and $S_{(3.4)}$ is the asymptotic solution by formula (3.4). One can see that the relative error for the $E_{1}$ component is maximal for $\zeta=100$ and is equal to $3.5 \%$ when $r / a=5.0$. The error for $\zeta=100$ decays fast as $r / a$ grows and is equal to $0.3 \%$ at $r / a=20.0$. The relative error decays slower for larger values of $\zeta$. Nevertheless, the relative error for $\zeta=500$ remains small: for all considered values of $r / a$ this error changes from $0.5 \%$ when $r / a=5.0$ to $0.3 \%$ when $r / a=20.0$.

The relative error for component $E_{2}$ practically does not depend on the $r / a$, except for $\zeta=500$. The maximal value of error is attained at $\zeta=20$, it is equal to $15.3 \%$ and it diminishes if $\zeta$ decreases. The minimal value of error for this component is attained for $\zeta=500$ at $r / a=20$ and it is equal to $0.6 \%$.

The relative error for the field component $E_{3}$ depends significantly on $\zeta$. The maximal value of this error is attained for $\zeta=500$ when $r / a=5.0$ and is equal to $2.3 \%$. The minimal value of the error is equal to $0.1 \%$ and is attained for $\zeta=100$ when $r / a=20.0$. Summarizing the above results, one concludes that 


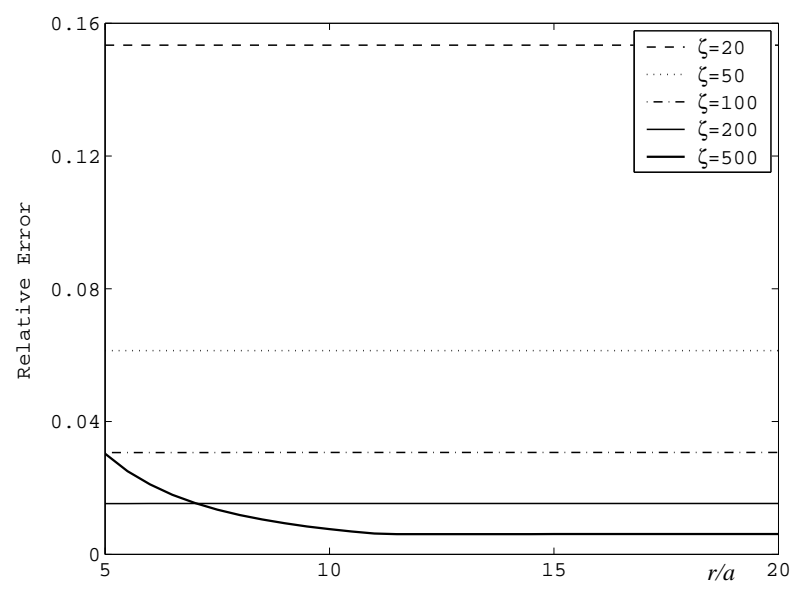

Figure 2. Relative error for $E_{2}$ component, $k=0.001$

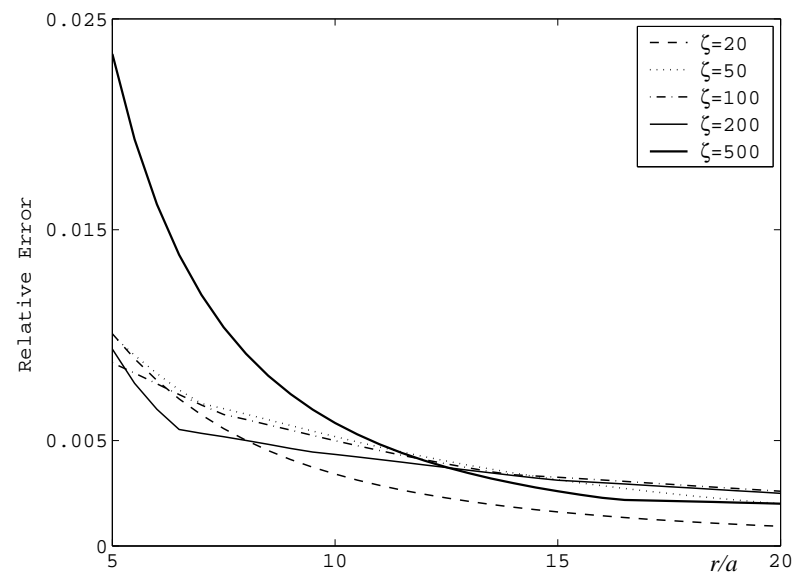

FiguRE 3. Relative error for $E_{3}$ component, $k=0.001$

the relative error of the asymptotical solution (3.4) when $r / a \geq 20.0$ does not exceed $1 \%$ for $E_{1}$ and $E_{3}$ components for all considered values of $\zeta$. The relative error for $E_{2}$ component when $r / a=20.0$ depends on $\zeta$ and varies from $15.3 \%$ to $1.2 \%$.

\subsection{Comparison with the Mie-type solution}

In the previous subsection, we have estimated the accuracy of formula (3.4) for the EM field in the far zone by comparing it with the exact formula (3.3). From the practical point of view it is of interest to compare the accuracy of the solutions (3.3) and (3.4) with the accuracy of the Mie-type solution obtained in paper [1]. In the far zone, this solution can be calculated by the following formula:

$$
\begin{gathered}
E(p)=e^{i p} \cos (\theta) e_{1}+2 i a_{1} \frac{e^{i p}}{p^{2}} \sin (\theta) \cos (\varphi) \widehat{e}_{r}+ \\
+\left(-b_{1} \frac{e^{i p}}{p} \cos (\varphi)+i a_{1} \frac{e^{i p}(1+i p)}{p 2} \cos (\theta) \cos (\varphi)\right) \widehat{e}_{\theta}+ \\
+\left(b_{1} \frac{e^{i p}}{p} \cos (\theta) \sin (\varphi)-i a_{1} \frac{e^{i p}(1+i p)}{p 2} \sin (\varphi)\right) \widehat{e}_{\varphi}
\end{gathered}
$$




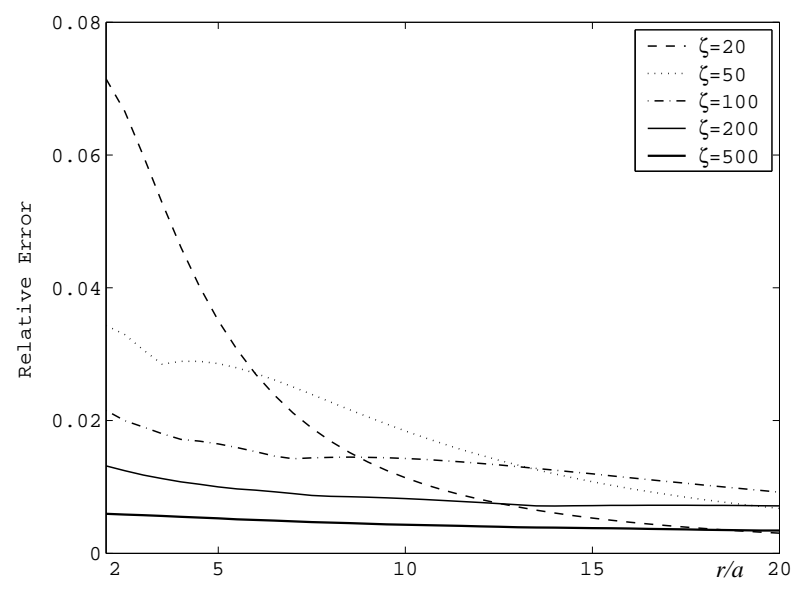

FiguRE 4. Relative error of (3.3) for $E_{r}$ component, $k=0.001$

where $p=k r$ is distance to far zone. We will use formula (3.6) for comparison with solutions (3.3) and (3.4), because it was established in [1] that the relative error of formula (3.6) does not exceed $0.1 \%$ for all values of parameters $k, a, r$, and $\zeta$ used below. This was established by comparing the numerical results, obtained by formula (3.6) with the exact solution, given in [1], see formulas (43)-(44) in [1]. Since the solution (3.6) is given in the spherical coordinates, we use the formulas for the electric field also in the spherical coordinates:

$$
\begin{gathered}
E_{r}=E_{1} \sin (\theta) \cos (\varphi)+E_{2} \sin (\theta) \sin (\varphi)+E_{3} \cos (\theta) \\
E_{\theta}=E_{1} \cos (\theta) \cos (\varphi)+E_{2} \cos (\theta) \sin (\varphi)-E_{3} \sin (\theta), \\
E_{\varphi}=-E_{1} \sin (\varphi)+E_{2} \cos (\varphi)
\end{gathered}
$$

In Fig. 4, the results of the comparison of the solution, obtained by formulas (3.3) and (3.6) are given for the $E_{r}$ component. The dependence of the relative error on the value of the parameter $r / a$ is shown. Solution (3.6) is considered here as the benchmark (exact) solution. The relative error depends on $r / a$ and $\zeta$. For example, when $r / a=2.0$ and $\zeta=20$, then the relative error is equal to $7.1 \%$, if $r / a$ grows this error decays and does not exceed $0.9 \%$ when $r / a=20.0$ for all considered values of $\zeta$. The relative error for $E_{\varphi}$ component is $7.1 \%$ when $r / a=2.0$ and is not more than $0.2 \%$ at $r / a=20.0$ for all considered values of $\zeta$. The relative error of formula (3.4) in the far zone is about the same as the relative error of formula (3.3). The comparison is made using formula (3.6) in the far zone.

In Figs. 5-7, the relative error of the solution, calculated by formula (3.4) is given. The Mie-type solution (3.6) is considered as the exact solution. The results are shown for the components $E_{r}, E_{\theta}$, and $E_{\varphi}$, respectively. The relative error for the $E_{r}$ component is maximal when $\zeta=500$ and $r / a=2.0$ : this error is equal to $0.2 \%$. The maximal relative error when $r / a=20.0$ and $\zeta=500$ is equal to $0.02 \%$.

The relative error for the $E_{\theta}$ component (see Fig. 6) practically does not depend on $r / a$ and $\zeta$ and is equal to $1.23 \%$. The relative error for the $E_{\varphi}$ component (see Fig. 7 ) does not exceed $0.01 \%$ for all considered values of $r / a$ and $\zeta$. The relative error depends on the value of $k$ for the considered range of $r / a$. In Fig. 8, the relative error for the $E_{r}$ component is shown for $k=0.5$. This error is about $40 \%$ when $r / a=2.0$ and $\zeta=500$, it decays fast when $r / a$ grows for all considered values of $\zeta$ and it it is less than $3.6 \%$ when $r / a=20.0$ and $\zeta=500$. The minimal value of the error for $r / a=20.0$ is attained for $\zeta=20$ and is equal to $0.1 \%$. The relative error for the $E_{\theta}$ component has the same order as the $E_{r}$ component, and the relative error for the $E_{\varphi}$ component changes from $9.3 \%$ to $0.9 \%$ for the considered values of $r / a$ and $\zeta$. The above numerical results show that the accuracy of solution (2.29) increases as $k a$ decreases. 


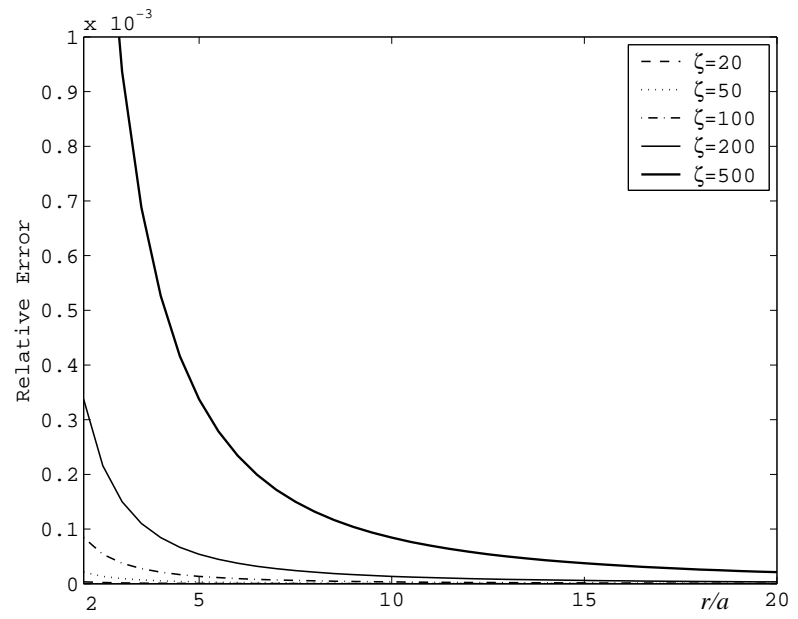

FiguRE 5. Relative error of (3.4) for $E_{r}$ component, $k=0.001$

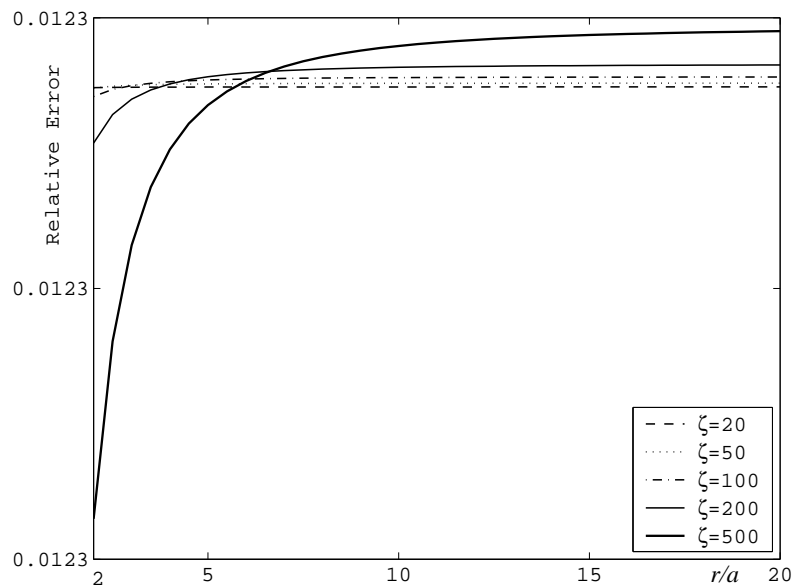

FiguRE 6. Relative error of (3.4) for $E_{\theta}$ component, $k=0.001$

The numerical results related to investigation of the dependence of relative error for the $E_{r}$ component in formula (3.6) on $\zeta$ at fixed $r$ are shown in Fig. 9. Solution (3.6) is considered as the benchmark solution. The relative error grows as $\zeta$ increases. When $\zeta=20$ the maximal relative error is attained at $r=5.0$ and it is equal to $0.01 \%$. When $\zeta=500$ the maximal relative error is attained at $r=5.0$ and it is equal to $3.4 \%$. The minimal error for $\zeta=500$ is attained when $r=20.0$ and it is equal to $0.2 \%$. The calculations show that error diminishes when $k$ decreases. When $k=0.001$ the maximal error for $r=5.0$ and $\zeta=500$ is equal to $0.3 \%$. This error for $r=20.0$ is equal to $0.05 \%$. These results show that the EM field for the far zone given by formula (3.4) are very close to the benchmark (exact) results obtained by using the Mie-type solution (3.6) in the far zone. The maximal relative error does not exceed $3.5 \%$ for the considered range of the parameters $k, a, r$, and $\zeta$. 


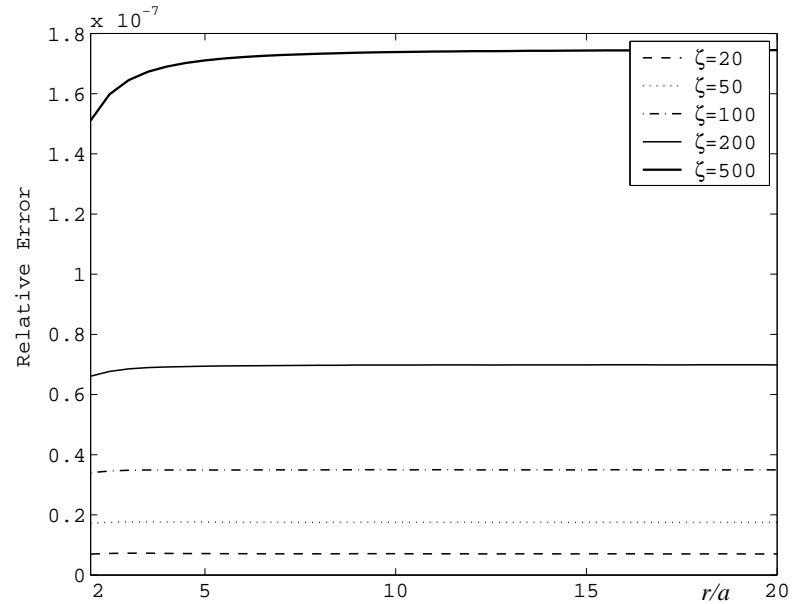

FiguRE 7. Relative error of (3.4) for $E_{\varphi}$ component, $k=0.001$

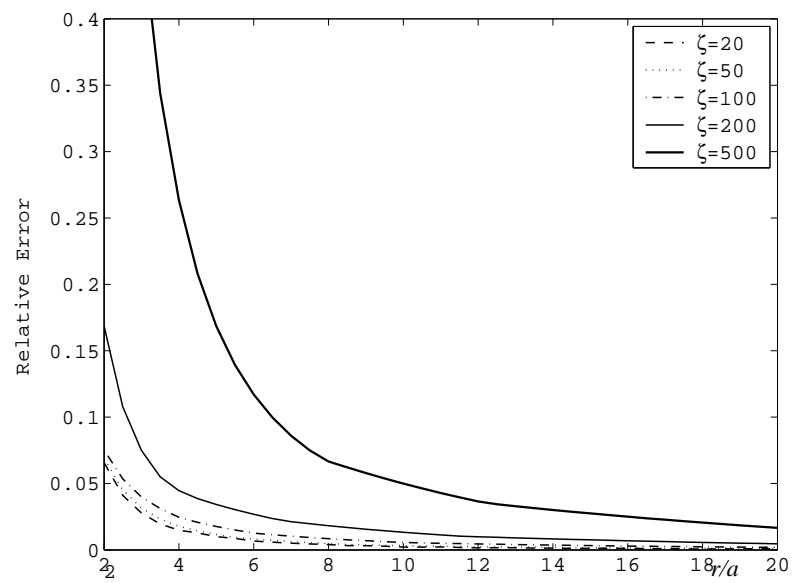

FiguRE 8. Relative error of (3.4) for $E_{r}$ component, $k=0.5$

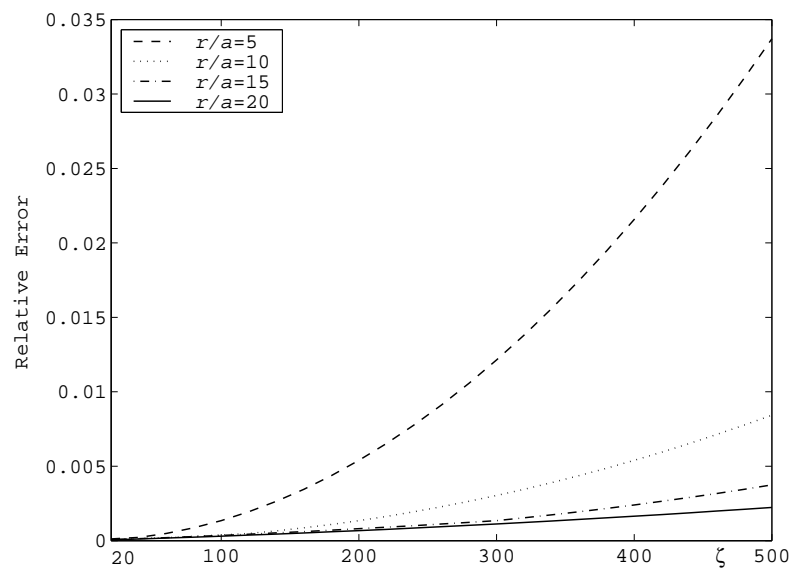

Figure 9. Relative error of (3.4) for $E_{r}$ component, $k=0.1$ 


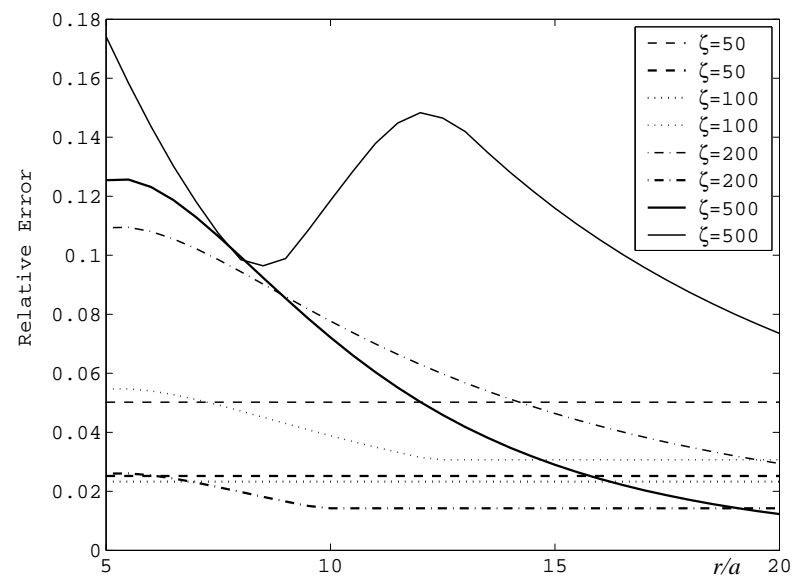

FiguRE 10. Relative errors of (3.3) and (3.8) for $E_{r}$ component

\subsection{Comparative results on the accuracy of formula (3.3)}

In paper [1], the numerical experiments related to investigation of the accuracy of formula (53) were given. Formula (53) from [1] looks as follows:

$$
E(x)=E_{0}(x)-\frac{8 a^{2} \pi i \zeta}{3 \omega \mu_{0}}\left[\nabla_{x} g(x, O), \nabla \times E_{0}(O)\right] .
$$

Formula (3.3) is more accurate than (3.8) because it is based on a theoretically exact argument and, because of this, it contains the additional term $\tau$, which was neglected in [1]. We give below the relative error of both formulas for various $\zeta$ in order to explain the advantages of the new formula (3.3) over formula (3.8).

The Mie-type solution (3.6) is considered as the benchmark solution. The results are shown in Figs. 10-12 for the components $E_{r}, E_{\theta}$, and $E_{\varphi}$, respectively, $k=0.5, a=0.1$. The thin lines correspond to the solution (3.8) from [1], and the thick ones correspond to the solution (3.3). One can see from Fig. 10, that the maximal decrease of the relative error for $E_{r}$ component is observed at $r / a=20.0$ for $\zeta=500$, the error decreases from $7.3 \%$ to $1.2 \%$. Considerable decrease of the error is observed for $\zeta=200$ : the error decreases from $10.9 \%$ to $2.6 \%$ at $r / a=5.0$ and it decreases from $2.9 \%$ to $1.4 \%$ when $r / a=20.0$. For $\zeta=50$ and $\zeta=100$ the relative error practically does not depend on $r / a$ and decreases not as much. The comparison of the relative errors for the $E_{\theta}$ component shows that the maximal decrease of this error is observed for $\zeta=500$ : the relative error decreases from $40.2 \%$ to $8.1 \%$ when $r / a=5.0$, and it decreases from $3.7 \%$ to $1.1 \%$ when $r / a=20.0$. For $\zeta=200$ the relative error decreases from $19.4 \%$ to $1.5 \%$ when $r / a=5.0$, and from $1.8 \%$ to $1.2 \%$ when $r / a=20.0$. For $\zeta=50$ and $\zeta=100$, the relative error for the $E_{\theta}$ component in solution (3.8) depends on relation $r / a$ slightly and it decreases from $7.2 \%$ up to $6.9 \%$ and from $9.7 \%$ up to $3.5 \%$, respectively. For solution (3.3), this decrease amounts from $5.9 \%$ up to $5.6 \%$ and from $3.1 \%$ up to $2.8 \%$, respectively. The relative errors for the $E_{\varphi}$ component behave similarly. The maximal decrease of the relative error is observed for $\zeta=500$ : this error decreases from $13.5 \%$ to $2.1 \%$ when $r / a=5.0$ and it decreases from $0.7 \%$ to $0.01 \%$ when $r / a=20.0$. For $\zeta=200$, the error decreases from $5.4 \%$ to $0.3 \%$ when $r / a=5.0$ and from $0.3 \%$ to $0.08 \%$ when $r / a=20.0$. For $\zeta=50$ and $\zeta=100$ the relative error of the new solution (3.3) does not exceed $0.34 \%$. These results show that the new solution (3.3) is much more accurate than the solution (3.8) from [1]. 


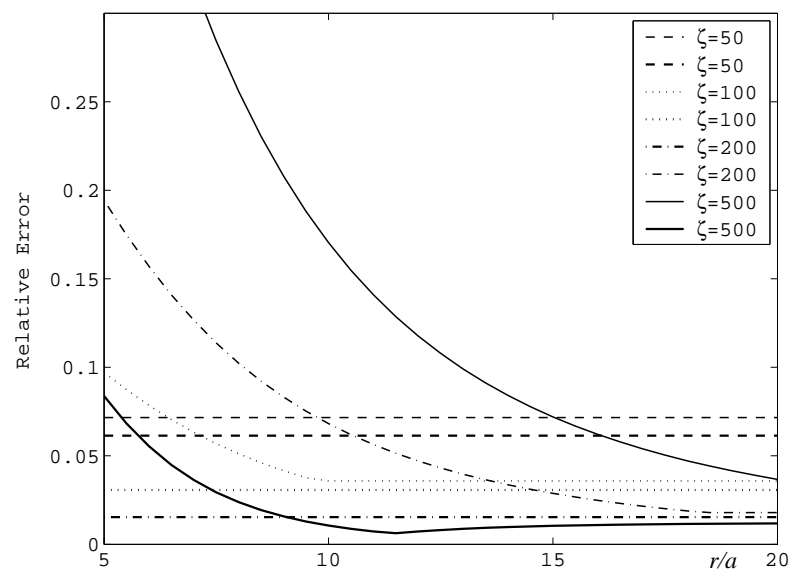

Figure 11. Relative errors of (3.3) and (3.8) for $E_{\theta}$ component

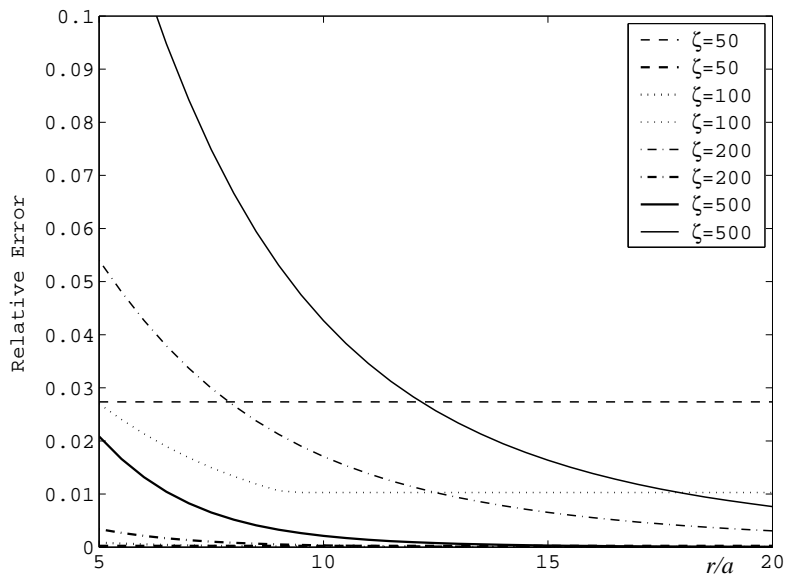

Figure 12. Relative errors of (3.3) and (3.8) for $E_{\varphi}$ component

\section{Conclusions}

The explicit analytical formulas (2.29) and (2.30) for the EM field components are derived and tested numerically. To establish the limits of the applicability of the new formulas they are compared numerically with the Mie-type solution, obtained in [1] for a spherical particle and the impedance boundary condition. This solution is used as the benchmark (exact) solution for comparison with the new formulas (2.29) and (2.30), and with their versions (3.3) and (3.4), used in computations.

Comparison of formula (3.3) for the EM field components and its representation (3.4) for the far zone demonstrates that formula (3.4) yields the relative error not exceeding $2.5 \%$ when $r / a \geq 15.0$ for all the values of $\zeta$ in the range from 20 to 100. This error is larger at the smaller values of $r / a$ as $\zeta$ increases and it grows slightly at greater $r / a$ if $\zeta$ increases. Formulas (3.4) and (3.6) representing the new solution in the far zone and the Mie-type solution yield very similar numerical results. The relative error of formula (3.4) does not exceed $0.012 \%$ for the considered range of parameters $k, a, r$, and $\zeta$. Therefore, formula (3.4) is applicable in a wide range of parameters. The numerical results show that formula (3.3) is much more accurate than formula (3.8). This is a consequence of an exact theoretical derivation, which took into account the additional parameter $\tau$, which was neglected in [1]. 


\section{References}

[1] M. I. Andriychuk, S. W. Indratno, A. G. Ramm. Electromagnetic wave scattering by a small impedance particle: theory and modeling. Optics Communications, 285 (2012), 1684-1691.

[2] L. Landau, L. Lifshitz. Electrodynamics of continuous media. Pergamon Press, London, 1982.

[3] A.G.Ramm, Scattering of Acoustic and Electromagnetic Waves by Small Bodies of Arbitrary Shapes. Applications to Creating New Engineered Materials, Momentum Press, New York, 2013.

[4] A. G. Ramm. Many-body wave scattering by small bodies and applications. J. Math. Phys., 48 (2007), no. $10,103511$.

[5] A. G. Ramm. Wave scattering by many small particles embedded in a medium. Phys. Lett. A, 372/17 (2008), 3064-3070.

[6] A. G. Ramm. Distribution of particles which produces a "smart" material. Jour. Stat. Phys., 127 (2007), no. 5, 915-934.

[7] A. G. Ramm. Scattering by many small bodies and applications to condensed matter physics. Europ. Phys. Lett., 80 (2007), 44001.

[8] A. G. Ramm. Distribution of particles which produces a desired radiation pattern. Physica B, 394 (2007), no. 2, $253-255$.

[9] A. G. Ramm. A recipe for making materials with negative refraction in acoustics. Phys. Lett. A, $372 / 13$ (2008), $2319-2321$.

[10] A. G. Ramm. Does negative refraction make a perfect lens? Phys. Lett. A, 372 (2008), 6518-6520.

[11] A. G. Ramm. Electromagnetic wave scattering by small bodies. Phys. Lett. A, 372/23 (2008), 4298-4306.

[12] A. G. Ramm. Preparing materials with a desired refraction coefficient and applications, In the book "Topics in Chaotic Systems: Selected Papers from Chaos 2008 International Conference”. Editors C.Skiadas, I. Dimotikalis, Char. Skiadas, World Sci.Publishing, (2009), 265-273.

[13] A. G. Ramm. Materials with a desired refraction coefficient can be created by embedding small particles into the given material. International Journal of Structural Changes in Solids (IJSCS), 2 (2010), no. 2, 17-23.

[14] A. G . Ramm. Wave scattering by many small bodies and creating materials with a desired refraction coefficient. Afrika Matematika, 22 (2011), no. 1, 33-55.

[15] A. G. Ramm, Scattering by many small inhomogeneities and applications. In the book "Topics in Chaotic Systems: Selected Papers from Chaos 2010 International Conference", Editors C.Skiadas, I. Dimotikalis, Char. Skiadas, World Sci.Publishing, (2011), 41-52.

[16] A. G. Ramm. Electromagnetic wave scattering by many small bodies and creating materials with a desired refraction coefficient. Progress in Electromagnetic Research M (PIER M), 13 (2010), 203-215.

[17] A. G. Ramm. Wave scattering by small bodies of arbitrary shapes. World Sci. Publishers, Singapore, 2005.

[18] A. G. Ramm. Scattering by obstacles. D.Reidel, Dordrecht, 1986.

[19] A. G. Ramm. Electromagnetic wave scattering by a small impedance particle of an arbitrary shape. Optics Communications, 284 (2011), 3872-3877. 